Research Article

\title{
Network Pharmacology Reveals the Molecular Mechanism of Cuyuxunxi Prescription in Promoting Wound Healing in Patients with Anal Fistula
}

\author{
Yin Qu $\mathbb{D}$, Zhijun Zhang, Yafeng Lu, De Zheng, and Yang Wei $\mathbb{C}$ \\ Department of Anorectal Surgery, Shuguang Hospital, Shanghai University of Traditional Chinese Medicine, \\ Shanghai 201210, China \\ Correspondence should be addressed to Yang Wei; yangweiyishi@163.com
}

Received 15 May 2019; Revised 29 July 2019; Accepted 6 August 2019; Published 17 September 2019

Academic Editor: Caigan Du

Copyright $(2019$ Yin Qu et al. This is an open access article distributed under the Creative Commons Attribution License, which permits unrestricted use, distribution, and reproduction in any medium, provided the original work is properly cited.

Background. The healing process of the surgical wound of anal fistulotomy is much slower because of the presence of stool within the wound. Cuyuxunxi (CYXX) prescription is a Chinese herbal fumigant that is being used to wash surgical wound after anal fistulotomy. This study aimed at investigating the molecular mechanism of CYXX prescription using a network pharmacologybased strategy. Materials and Methods. The active compounds in each herbal medicine were retrieved from the traditional Chinese medicine systems pharmacology (TCMSP) database and in Traditional Chinese Medicine Integrated Database (TCMID) analysis platform based on the criteria of oral bioavailability $\geq 40 \%$ and drug-likeness $\geq 0.2$. The disease-related target genes were extracted from the Comparative Toxicogenomics Database. Protein-protein interaction network was built for the overlapped genes as well as functional enrichment analysis. Finally, an ingredient-target genes-pathway network was built by integrating all information. Results. A total of 375 chemical ingredients of the 5 main herbal medicines in CYXX prescription were retrieved from TCMSP database and TCMID. Among the 375 chemical ingredients, 59 were active compounds. Besides, 325 target genes for 16 active compounds in 3 herbal medicines were obtained. Functional enrichment analysis revealed that these overlapped genes were significantly related with immune response, biosynthesis of antibiotics, and complement and coagulation cascades. A comprehensive network which contains 133 nodes ( 8 disease nodes, 3 drug nodes, 8 ingredients, 103 target gene nodes, 7 GO nodes, and 4 pathway nodes) was built. Conclusion. The network built in this study might aid in understanding the action mechanism of CYXX prescription at molecular level to pathway level.

\section{Introduction}

Anal fistula is characterized by chronic abnormal communication between the epithelialized surface of the anal canal and the perianal skin [1]. The incidence and epidemiology of anal fistula were estimated as 8.6 per 100,000 population for nonspecific fistula, with 12.3 per 100,000 for males and 5.6 for females [2]. The incidence of anal fistula is increasing annually in the European Union [3], which significantly affects patients' quality of life. Treatment of anal fistula is a challenge for physicians for thousand years. The most frequently used therapy for treating anal fistula is surgery, such as simple fistulotomy [4]. However, it is associated with a significant risk of recurrence and complications such as fecal incontinence $[5,6]$. Besides, the surgical wound infection is hard to healing and easy to occur because of its special location. Therefore, strategies for reducing the incidence of complications are urgently needed for physicians.

Traditional Chinese medicine (TCM) plays an important role in maintaining health for Asian people. TCM has attracted the most attention for western countries in these years because of its reliable therapeutic efficacy and fewer side effects $[7,8]$. In recent years, fumigation-washing therapy on the surgical wound using Chinese herbal fumigant has been widely used for postoperative treatment and achieved satisfied effect. Cuyuxunxi (CYXX) prescription is a Chinese herbal fumigant composed of Reynoutria japonica Houtt. (30 g), Taraxacum mongolicum Hand.-Mazz. (30 g), 
Rhus chinensis Mill. (15g), Sophora flavescens Aiton (9g), and Angelica sinensis (Oliv.) Diels (9g). Our clinical studies have proved that long-time treatment with a low concentration of CYXX prescription can relieve pain, pruritus, turgescence, and effusion after anal fistulotomy and promote surgical wound healing [9]. However, the underlying mechanisms have seldom investigated.

The molecular mechanism of TCM, especially TCM formulae, is difficult to investigate previously because of the complex chemical composition and complex interactions among multiple components. Network pharmacology is a new method based on the theory of network biology. It has made a significant contribution to investigate the molecular mechanisms of TCM by combining pharmacokinetic evaluation and bioinformatics. In the past decades, tens of studies have proven the feasibility of network pharmacology in investigating the molecular mechanism of TCM [10-13]. In this study, we aimed at investigating the mechanisms of CYXX prescription in promoting surgical wound healing using a comprehensive network pharmacology-based approach.

\section{Materials and Methods}

2.1. Search for Chemical Ingredients. The traditional Chinese medicine systems pharmacology database and analysis platform (TCMCP) (http://sm.nwsuaf.edu.cn/lsp/tcmsp. php) contains large number of herbal entries, drug-target networks, and drug-disease networks, which help researchers in revealing the mechanisms of action of Chinese herbs [14]. The information of each herbal medicine, including the ingredients, molecule name, molecular weight $(\mathrm{MW})$, water partition coefficient $(A \log P)$, number of hydrogen bond donors and receptors (Hdon/Hacc), human oral bioavailability (OB), half-life (HL), Caco-2 permeability (Caco-2), blood-brain barrier (BBB), and drug-likeness (DL), was obtained. For the herb whose information was not obtained from TCMCP database, we further searched its information in Traditional Chinese Medicine Integrated Database (TCMID) (http://www.megabionet.org/tcmid/), which is a comprehensive database aiming at TCM's modernization and standardization [15].

2.2. Screening for Active Compounds. The active compounds were screened out on the basis of the ADME processes. The parameters of ADME include OB, DL, and HL. In this study, the compounds with $\mathrm{OB} \geq 40 \%$ and $\mathrm{DL} \geq 0.2$ were regarded as active compounds.

2.3. Identification of Target Proteins of Active Compounds. DrugBank database (http://www.drugbank.ca) is a unique bioinformatics and cheminformatics resource that combines detailed drug data with comprehensive drug-target information $[16,17]$. We searched the direct target proteins of each chemical ingredient in CYXX prescription from the DrugBank database. The full name of the target proteins was converted to gene symbol based on the UniProt ID in the
UniProt database (https://www.uniprot.org/) for further analysis.

2.4. Screening of Disease-Related Genes. Comparative Toxicogenomics Database (CTD, http://ctdbase.org/) is a crossspecies resource for building chemical-gene interaction networks $[18,19]$. This database facilitates identification and understanding of chemical-gene-disease associations [20]. We searched the disease-related genes in CTD using the keywords of "acute pain," "edema," "angioedema," "surgical wound dehiscence," "surgical wound infection," "wound infection," "skin ulcer," "pyoderma gangrenosum," "cutaneous fistula," "fissure in Ano," "fasciitis," and "Necrotizing." The intersection between the disease-related genes obtained from CTD and the target genes of active compounds was reserved for further analysis.

2.5. Functional Enrichment Analysis. The Database for Annotation, Visualization, and Integrated Discovery (DAVID, https://david.ncifcrf.gov/) v6.8 provides a comprehensive set of functional annotation tools for investigators to understand biological meaning behind large list of genes. In order to investigate the functions of the target genes, we performed gene ontology (GO) biological process (BP) enrichment analysis and Kyoto Encyclopedia of Genes and Genomes (KEGG) pathway enrichment analysis using DAVID. $P$ value $<0.05$ was regarded as the significance cutoff in this study.

2.6. Protein-Protein Interaction (PPI) Network Construction. STRING database contains all publicly available sources of PPI information [21]. The interactions of the overlapped target genes were predicted using the STRING database based on the cutoff criterion of required confidence $>0.4$. The PPIs satisfied this criterion were downloaded and submitted to Cytoscape software [22] (version 3.6.1, Boston, MA, USA) for visualizing the PPI network.

2.7. Construction of Ingredient-Target Genes-Pathway Network. The predicting target genes of each ingredient of CYXX prescription, the disease-related genes, and the GOBP or KEGG pathway involved were connected to build an integrated network to illustrate the pharmacological mechanisms of CYXX prescription. The graphical interaction of this network was visualized by Cytoscape software.

\section{Results}

3.1. Identification of Target Genes of CYXX Prescription. The CYXX prescription consists of 5 main herbal medicines, Sophorae Flavescentis Radix, Polygoni Cuspidati Rhizoma Radix, Angelicae Sinensis Radix, Galla Chinensis, and Taraxacum mongolicum. We searched these keywords in TCMSP database. A total of $113,62,125,3$, and 0 chemical ingredients of the five herbal medicines in CYXX prescription were retrieved from TCMSP database. Since there 
was no result for Taraxacum mongolicum in TCMSP, we further searched its chemical ingredients in TCMID using the keyword of "PU GONG YING" and obtained 72 chemical ingredients. Based on the criteria of $\mathrm{OB} \geq 40 \%$ and $\mathrm{DL} \geq 0.2$, a total of $37,6,1,1$, and 14 active compounds were identified for these 5 herbal medicines finally (Table 1). A herb-active compounds network was constructed by using Cytoscape (Figure 1).

The direct target proteins of each chemical ingredient in CYXX prescription were searched from the DrugBank database, and 345 target genes for 16 active compounds in 3 herbal medicines were obtained (Supplementary Table 1).

3.2. Screening of Disease-Related Genes. Since the CYXX prediction is used for anti-infection and promoting wound healing after anal fistula surgery, we searched disease related with anti-infection and wound healing in CTD. The overlapped genes between the disease-related genes obtained from CTD and the target genes of active compounds were identified. Finally, we obtained a total of 313 overlapped genes between 8 disease-related genesets and the target genes of active compounds (Table 2).

\subsection{Functional Annotation Analysis of the Overlapped Genes.} GO-BP and KEGG pathway enrichment analyses were further performed for the overlapped genes. As shown in Table 3, these genes were significantly related with KEGG pathways of "complement and coagulation cascades" $(P=1.90 E-28)$, "biosynthesis of antibiotics" $(1.70 E-04)$, "HIF-1 signaling pathway" ( $P=5.15 E-04)$, and "antigen processing and presentation" $(P=0.02474)$. Besides, the GO-BP terms of "platelet degranulation" $(P=5.95 E-24)$, "complement activation, classical pathway" $(P=2.80 E-19)$, "regulation of complement activation" $(P=1.91 E-15)$, "complement activation" $(P=4.28 E-12)$, and "innate immune response" $(P=1.94 E-11)$ were significantly enriched.

3.4. Construction of Ingredient-Target Genes-Pathway Network. A comprehensive network contains 133 nodes (8 disease nodes, 3 drug nodes, 8 ingredients, 103 target gene nodes, 7 GO nodes, and 4 pathway nodes) was built by integrating all information (Figure 2). For all the 8 disease nodes, the degree of "skin ulcer" was the highest (degree $=103$ ) and the next one was "pyoderma gangrenosum" (degree $=30$ ). For the ingredients, zinc regulated the greatest number of target genes $($ degree $=60)$ and the subsequent one was copper (degree $=58$ ).

We further divided the comprehensive network into several subnetworks based on our interested pathways: hsa04610: complement and coagulation cascades, hsa01130: biosynthesis of antibiotics, hsa04066: HIF-1 signaling pathway, and hsa04612: antigen processing and presentation (Figure 3). From Figure 3(a), we could find that genes in the pathway of complement and coagulation cascades were closely related with the diseases of skin ulcer, surgical wound dehiscence, cutaneous fistula, acute pain, and pyoderma gangrenosum as well as the small molecules of zinc and copper. Both of these two small molecules were derived from Taraxacum mongolicum. From Figure 3(b), the small molecules of zinc, copper, and manganese derived from Taraxacum mongolicum were closely related with genes in the pathway of biosynthesis of antibiotics, which is a pathway that being disturbed in diseases of acute pain, skin ulcer, surgical wound dehiscence, pyoderma gangrenosum, and wound infection. From Figure 3(c), quercetin derived from Polygoni Cuspidati Rhizoma et Radix, Taraxacum mongolicum, and Sophorae Flavescentis Radix could regulate the expression of PI3KCG, which was dysregulated in skin ulcer. Besides, the small molecules iron, copper, zinc, vitamin C, manganese, and myristic acid from Taraxacum mongolicum could regulate the expression of SERPINE1, TFRC, EGLN1, and TLR4 in HIF-1 signaling pathway. These genes were frequently dysregulated in several diseases, including wound infection, skin ulcer, acute pain, surgical wound infection, cutaneous fistula, and pyoderma gangrenosum. In Figure 3(d), we could find that quercetin could regulate the expression of HSP90AA1 and HSPA2 in the pathway of antigen processing and presentation, which were dysregulated in diseases of skin ulcer and acute pain.

\section{Discussion}

TCM has been widely used in China for thousands of years. Though TCM has reliable therapeutic efficacy and fewer side effects, it is hard to be accepted by western countries because of its complex chemical composition and uncertain theory basis. The emergence of network pharmacology owning to the development of systems biology, bioinformatics, and polypharmacology has advanced the investigation of TCM into a new period [23-25]. By shifting from "one-target, onedrug" analysis to "network-targeted, multicomponent" analysis, network pharmacology is a powerful way for the molecular mechanism of TCM formula $[8,26]$.

In this study, we applied a network pharmacology-based strategy to investigate the molecular mechanism of CYXX prescription in relieving pain and promoting surgical wound healing after anal fistulotomy. A total of 375 chemical ingredients of the 5 main herbal medicines in CYXX prescription were retrieved from TCMSP database and TCMID. Among the 375 chemical ingredients, 59 active compounds that could overcome the barriers posed by absorption, distribution, metabolism, and excretion (ADME) processes were identified [27]. The recommended thresholds of $\mathrm{OB}$ and DL are $30 \%$ and 0.18 in TCMSP [14], respectively. In this study, we set the criteria of OB and DL as $40 \%$ and 0.20 , which were higher than the recommended threshold. These criteria were set based on our experience aimed at obtaining more reliable results. These data additionally indicate the potential of CYXX prescription as a promising therapy for surgical wound healing.

By searching the direct target proteins from the DrugBank database, 345 target genes for 16 active compounds in 3 herbal medicines were obtained. Meanwhile, target genes of 8 wound healing or anti-infection-related diseases were searched from CTD and the overlapped target genes between 
TABLE 1: The active compounds in the five main herbal medicines of Cuyuxunxi prescription.

\begin{tabular}{|c|c|c|c|c|c|c|c|c|}
\hline Herbs & Mol ID & $\begin{array}{c}\text { Molecule } \\
\text { name }\end{array}$ & MW & $A \log P$ & $\begin{array}{l}\mathrm{OB} \\
(\%)\end{array}$ & Caco-2 & $\mathrm{BBB}$ & $\mathrm{DL}$ \\
\hline \multirow{37}{*}{ Sophorae Flavescentis Radix } & MOL001040 & $\begin{array}{l}\text { (2R)-5,7-Dihydroxy-2-(4-hydroxyphenyl) } \\
\text { chroman-4-one }\end{array}$ & 272.27 & 2.3 & 42.36 & 0.38 & -0.48 & 0.21 \\
\hline & MOL001484 & Inermine & 284.28 & 2.44 & 75.18 & 0.89 & 0.4 & 0.54 \\
\hline & MOL003627 & Sophocarpine & 246.39 & 1.39 & 64.26 & 0.99 & 1 & 0.25 \\
\hline & MOL003648 & Inermin & 284.28 & 2.44 & 65.83 & 0.91 & 0.36 & 0.54 \\
\hline & MOL003673 & Wighteone & 338.38 & 3.92 & 42.8 & 0.64 & -0.16 & 0.36 \\
\hline & MOL003676 & Sophoramine & 244.37 & 1.15 & 42.16 & 1.43 & 1.53 & 0.25 \\
\hline & MOL003680 & Sophoridine & 248.41 & 1.42 & 60.07 & 1.13 & 1.14 & 0.25 \\
\hline & MOL000392 & Formononetin & 268.28 & 2.58 & 69.67 & 0.78 & 0.02 & 0.21 \\
\hline & MOL004580 & cis-Dihydroquercetin & 304.27 & 1.49 & 66.44 & -0.34 & -1.11 & 0.27 \\
\hline & MOL005100 & $\begin{array}{l}\text { 5,7-Dihydroxy-2-(3-hydroxy-4- } \\
\text { methoxyphenyl)chroman-4-one }\end{array}$ & 302.3 & 2.28 & 47.74 & 0.28 & -0.3 & 0.27 \\
\hline & MOL005944 & Matrine & 248.41 & 1.42 & 63.77 & 1.39 & 1.52 & 0.25 \\
\hline & MOL006562 & (+)-7,11-Dehydromatrine, (leontalbinine) & 246.39 & 1.42 & 62.08 & 1.06 & 1.12 & 0.25 \\
\hline & MOL006564 & $(+)$-Allomatrine & 248.41 & 1.42 & 58.87 & 1.08 & 1.13 & 0.25 \\
\hline & MOL006565 & AIDS211310 & 248.41 & 1.42 & 68.68 & 1.15 & 1.38 & 0.25 \\
\hline & MOL006566 & (+)-Lehmannine & 246.39 & 1.11 & 58.34 & 1.21 & 1.36 & 0.25 \\
\hline & MOL006568 & Isosophocarpine & 246.39 & 1.39 & 61.57 & 1.39 & 1.45 & 0.25 \\
\hline & MOL006571 & Anagyrine & 244.37 & 1.15 & 62.01 & 1.16 & 1.13 & 0.24 \\
\hline & MOL006573 & 13,14-Dehydrosophoridine & 246.39 & 1.39 & 65.34 & 1.06 & 1.11 & 0.25 \\
\hline & MOL006582 & $5 \alpha, 9 \alpha$-Dihydroxymatrine & 280.41 & -0.3 & 40.93 & 0.04 & -0.43 & 0.32 \\
\hline & MOL006583 & 7,11-Dehydromatrine & 246.39 & 1.42 & 44.43 & 1.11 & 1.13 & 0.25 \\
\hline & MOL006596 & Glyceollin & 338.38 & 2.85 & 97.27 & 0.53 & -0.19 & 0.76 \\
\hline & MOL003347 & Hyperforin & 536.87 & 8.62 & 44.03 & 0.87 & 0.4 & 0.6 \\
\hline & MOL006604 & $\begin{array}{l}\text { (2S)-7-Hydroxy-2-(4-hydroxyphenyl)-5- } \\
\text { methoxy-8-(3-methylbut-2-enyl)chroman- } \\
\text { 4-one }\end{array}$ & 354.43 & 4.41 & 48.09 & 0.8 & 0 & 0.39 \\
\hline & MOL006613 & Kushenin & 286.3 & 2.39 & 47.62 & 0.71 & 0.35 & 0.38 \\
\hline & MOL006619 & Kushenol J & 580.59 & -0.87 & 51.39 & -2.2 & -3.12 & 0.74 \\
\hline & MOL006620 & Kushenol J_qt & 286.3 & 2.27 & 50.86 & 0.24 & -0.27 & 0.24 \\
\hline & MOL006622 & Kushenol O & 562.57 & -0.56 & 42.41 & -1.67 & -2.25 & 0.76 \\
\hline & MOL006623 & Kushenol T & 442.55 & 4.46 & 51.28 & -0.05 & -0.95 & 0.64 \\
\hline & MOL006626 & Leachianone G & 356.4 & 3.89 & 60.97 & 0.33 & -0.36 & 0.4 \\
\hline & MOL006627 & Lehmanine & 246.39 & 1.11 & 62.23 & 1.18 & 1.25 & 0.25 \\
\hline & MOL006628 & (+)-Lupanine & 248.41 & 1.42 & 52.71 & 1.16 & 1.19 & 0.24 \\
\hline & MOL006630 & Norartocarpetin & 286.25 & 2.07 & 54.93 & 0.14 & -0.74 & 0.24 \\
\hline & MOL000456 & Phaseolin & 322.38 & 3.46 & 78.2 & 1.09 & 0.39 & 0.73 \\
\hline & MOL006649 & Sophranol & 264.41 & 0.67 & 55.42 & 0.6 & 0.68 & 0.28 \\
\hline & MOL006650 & $\begin{array}{c}\text { (-)-Maackiain-3-O-glucosyl-6'-O- } \\
\text { malonate }\end{array}$ & 532.49 & 0.7 & 48.69 & -1.45 & -2.14 & 0.52 \\
\hline & MOL006652 & Trifolrhizin & 462.44 & 0.95 & 48.53 & -0.85 & -1.65 & 0.74 \\
\hline & MOL000098 & Quercetin & 302.25 & 1.5 & 46.43 & 0.05 & -0.77 & 0.28 \\
\hline \multirow{6}{*}{$\begin{array}{l}\text { Polygoni Cuspidati Rhizoma et } \\
\text { Radix }\end{array}$} & MOL013288 & Picralinal & 366.45 & 1.8 & 58.01 & 0.23 & -0.21 & 0.75 \\
\hline & MOL002259 & Physcion diglucoside & 608.6 & -0.91 & 41.65 & -2.64 & -3.43 & 0.63 \\
\hline & MOL002268 & Rhein & 284.23 & 1.88 & 47.07 & -0.2 & -0.99 & 0.28 \\
\hline & MOL002280 & $\begin{array}{c}\text { Torachrysone-8-O-beta-D-( } 6^{\prime} \text {-oxayl)- } \\
\text { glucoside }\end{array}$ & 480.46 & 0.64 & 43.02 & -1.23 & -1.84 & 0.74 \\
\hline & MOL000492 & $(+)$-Catechin & 290.29 & 1.92 & 54.83 & -0.03 & -0.73 & 0.24 \\
\hline & MOL000098 & Quercetin & 302.25 & 1.5 & 46.43 & 0.05 & -0.77 & 0.28 \\
\hline Angelicae Sinensis Radix & MOL000449 & Stigmasterol & 412.77 & 7.64 & 43.83 & 1.44 & 1 & 0.76 \\
\hline Galla Chinensis & MOL000569 & Digallate & 322.24 & 1.53 & 61.85 & -0.76 & -1.52 & 0.26 \\
\hline
\end{tabular}


TABLE 1: Continued.

\begin{tabular}{|c|c|c|c|c|c|c|c|c|}
\hline Herbs & Mol ID & $\begin{array}{c}\text { Molecule } \\
\text { name }\end{array}$ & MW & $A \log P$ & $\begin{array}{l}\text { OB } \\
(\%)\end{array}$ & Caco-2 & $\mathrm{BBB}$ & $\mathrm{DL}$ \\
\hline $\begin{array}{l}\text { Taraxacum } \\
\text { mongolicum }\end{array}$ & $\begin{array}{c}1 \\
2 \\
3 \\
4 \\
5 \\
6 \\
7 \\
8 \\
9 \\
10 \\
11 \\
12 \\
13 \\
14\end{array}$ & $\begin{array}{c}\text { Calcium } \\
\text { Choline } \\
\text { Copper } \\
\text { Inulin } \\
\text { Iron } \\
\text { Manganese } \\
\text { Myristic acid } \\
\text { Palmitic acid } \\
\text { Potassium } \\
\text { Quercetin } \\
\text { Rutin } \\
\text { Stearic acid } \\
\text { Vitamin C } \\
\text { Zinc }\end{array}$ & & & & & & \\
\hline
\end{tabular}

MW, molecular weight; $A \log P$, water partition coefficient; OB, human oral bioavailability; Caco-2, Caco-2 permeability; BBB, blood-brain barrier; DL, druglikeness.

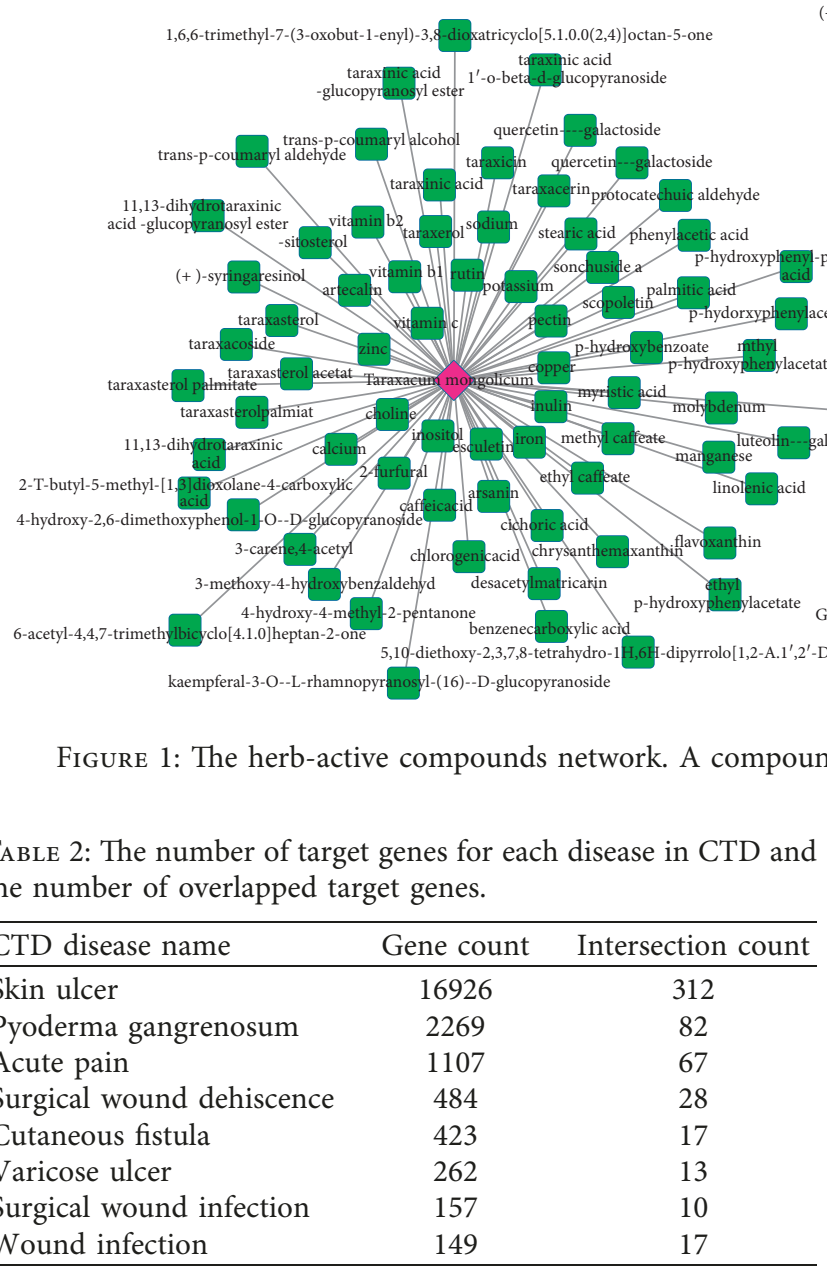

these two datasets were selected as candidates for further analysis. Compared to the surgical wound on other position, the healing process of the surgical wound of anal fistulotomy is much slower because of the presence of stool within the wound [5]. CYXX prescription is a TCM formula based on several years of clinical experiences. This prescription has been used for postoperative treatment for patients underwent anal fistulotomy. Results suggested that this prescription could relieve pain, pruritus, turgescence, and effusion and promote surgical wound healing [9]. Therefore, we speculated the molecular mechanism of CYXX prescription might be related with anti-infection. Therefore, diseases related with "pain," "turgescence," "edema" were searched from CTD including "acute pain," "edema," "angioedema," "surgical wound dehiscence," "surgical wound infection," "wound infection," "skin ulcer," "pyoderma gangrenosum," "cutaneous fistula," "fissure in Ano," "fasciitis," and "Necrotizing." Finally, 313 target genes of 8 diseases were obtained. 


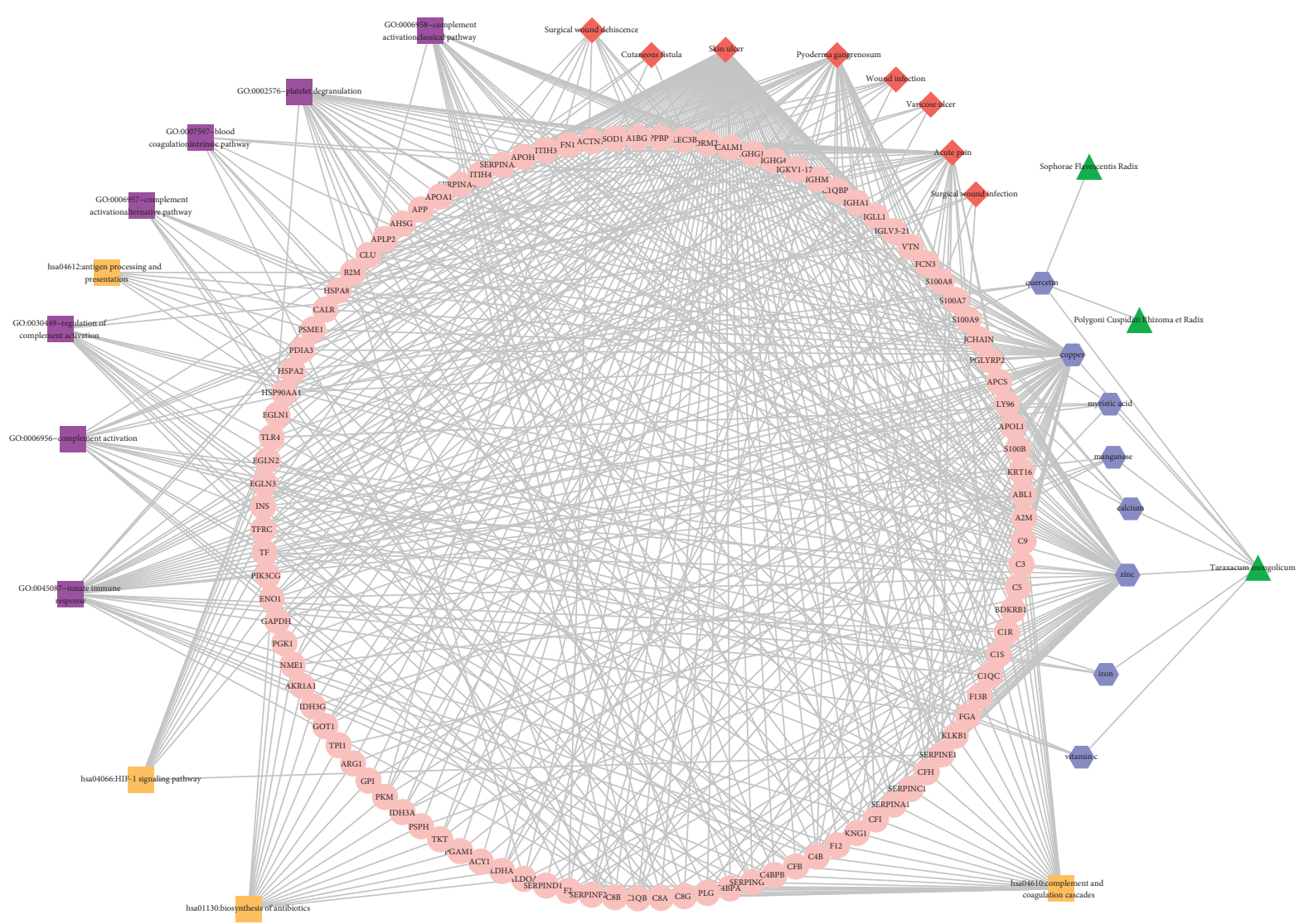

FIGURE 2: The ingredient-target genes-pathway network.

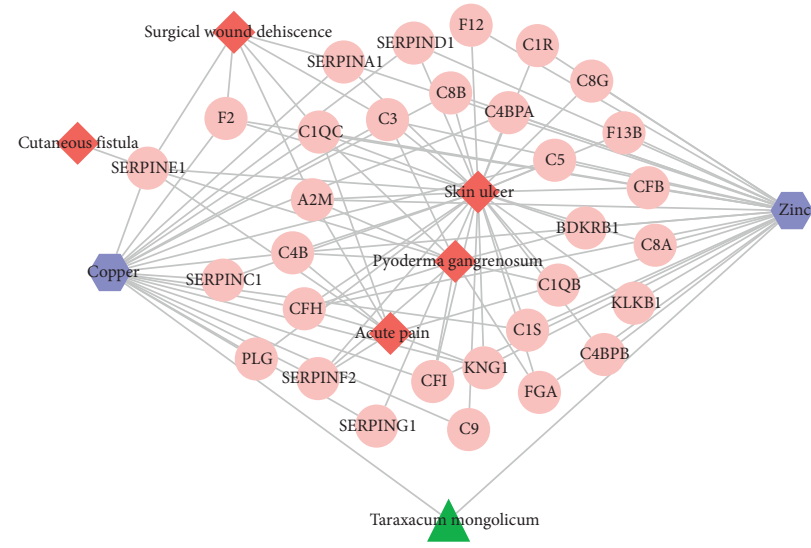

(a)

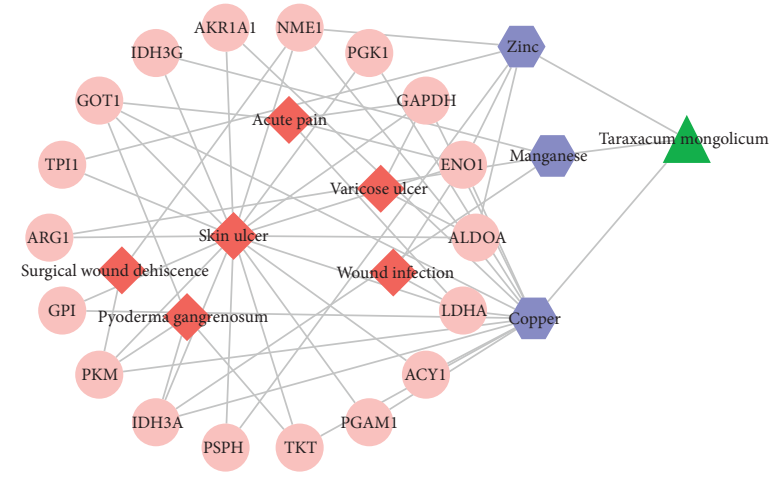

(b)

FIgUre 3: Continued. 


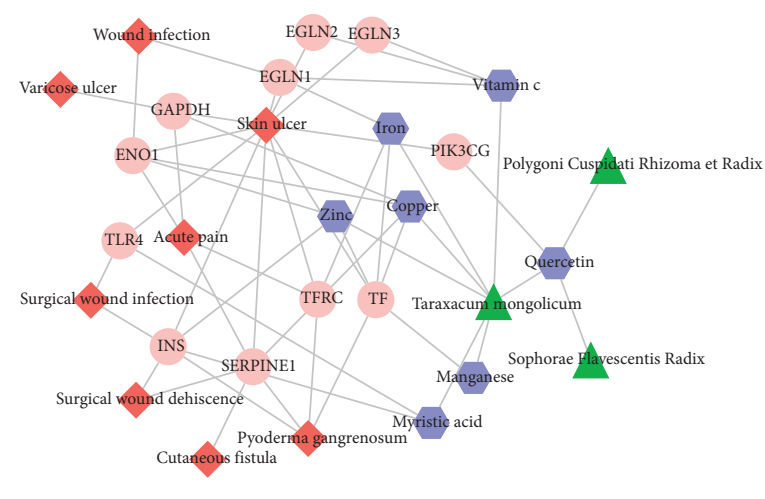

(c)

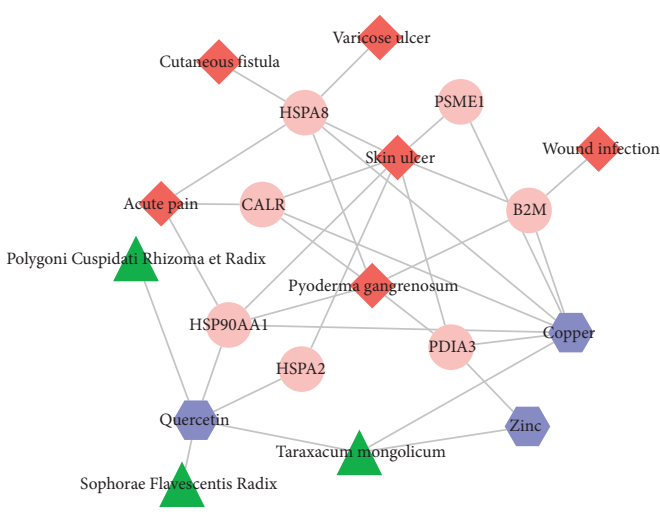

(d)

Figure 3: Subnetwork analysis.

TABle 3: The functional enrichment results for the overlapped target genes.

\begin{tabular}{lccc}
\hline Category & Term & Count & $P$ value \\
\hline KEGG & hsa04610: complement and coagulation cascades & 31 & $1.90 E-28$ \\
KEGG & hsa01130: biosynthesis of antibiotics & 18 & 11 \\
KEGG & hsa04066: HIF-1 signaling pathway & 7 & $5.70 E-04$ \\
KEGG & hsa04612: antigen processing and presentation & 28 & 0.02474 \\
GO BP & GO:0002576 platelet degranulation & 24 & $5.95 E-24$ \\
GO BP & GO:0006958 complement activation, classical & 24 & 14 \\
GO BP & gOthway $0030449 \sim$ regulation of complement activation & 17 & $1.91 E-15$ \\
GO BP & GO:0006956 complement activation & 33 & $4.28 E-12$ \\
GO BP & GO:0045087 innate immune response & 8 & $1.94 E-11$ \\
GO BP & GO:0006957 complement activation, alternative & pathway & 8 \\
GO BP & GO:0007597 blood coagulation, intrinsic pathway & $1.04 E-09$ \\
\hline
\end{tabular}

GO-BP and KEGG pathway enrichment analysis revealed that these overlapped genes were significantly related with immune response, biosynthesis of antibiotics, and complement and coagulation cascades. In the comprehensive ingredient-target genes-pathway network, we identified several active compounds in CYXX prescription that could act on these pathways. For example, genes in the pathway of complement and coagulation cascades were closely related with the diseases of skin ulcer, surgical wound dehiscence, cutaneous fistula, acute pain, and pyoderma gangrenosum as well as the small molecules of zinc and copper. Both of these two small molecules were derived from Taraxacum mongolicum. The complement system, as part of innate immunity, is activated immediately after trauma [28]. The complement system plays a central role in innate immunity, and the coagulation system is the main column in hemostasis which undergoes massive activation early after injury [29]. The humoral serine proteases in this pathway play central roles during the events of an inflammatory response [30]. In our subnetwork, serine proteases in coagulation and complement system, including SERPINE1, SERPINA1, SERPIND1, SERPINC1, SERPINF2, and SERPING1, are target genes of copper and zinc, which were derived from Taraxacum mongolicum. Therefore, we hypothesized that
Taraxacum mongolicum in CYXX prescription contributes to the role of relieving pain and promoting wound healing. These results further confirmed the reliability of our analysis and the role of CYXX prescription in clinic.

Despite the significant new findings of this study, some limitations still exist. This study is a primary study for investigating the molecular mechanism of CYXX prescription based on network pharmacology. Though this method has been proven effective by most studies, experimental validation for the network is still warranted for our study.

In conclusion, our results provide the theory basis for CYXX prescription in reducing complications and promoting wound healing after anal fistulotomy. The network built in this study might shed some new lights on understanding the pathogenesis of postoperative complications and aid in understanding the action mechanism of CYXX prescription at molecular level to pathway level. However, further experiments are warranted to validate the relationships of the network in this study.

\section{Data Availability}

The data used to support the findings of this study are available from the corresponding author upon request. 


\section{Disclosure}

Yin Qu and Zhijun Zhang should be regard as the co-first authors.

\section{Conflicts of Interest}

The authors declare that they have no conflicts of interest.

\section{Acknowledgments}

This work was supported by the Shanghai University of Traditional Chinese Medicine budget item (Program nos. 18LK039 and 2019LK014).

\section{Supplementary Materials}

The direct target proteins of chemical ingredients of the five herbal medicines in CYXX prescription in the DrugBank database. (Supplementary Materials)

\section{References}

[1] S. T. Feng, M. Huang, Z. Dong et al., "MRI T2-weighted imaging and fat-suppressed T2-weighted imaging image fusion technology improves image discriminability for the evaluation of anal fistulas," Korean Journal of Radiology, vol. 20, no. 3, pp. 429-437, 2019.

[2] P. Sainio, "Fistula-in-ano in a defined population. Incidence and epidemiological aspects," Annales Chirurgiae et Gynaecologiae, vol. 73, no. 4, pp. 219-224, 1984.

[3] C. Zanotti, C. Martinez-Puente, I. Pascual, M. Pascual, D. Herreros, and D. Garcia-Olmo, "An assessment of the incidence of fistula-in-ano in four countries of the European Union," International Journal of Colorectal Disease, vol. 22, no. 12, pp. 1459-1462, 2007.

[4] A. I. Malik and R. L. Nelson, "Surgical management of anal fistulae: a systematic review," Colorectal Disease, vol. 10, no. 5, pp. 420-430, 2008.

[5] H. Dudukgian and H. Abcarian, "Why do we have so much trouble treating anal fistula?," World Journal of Gastroenterology, vol. 17, no. 28, pp. 3292-3296, 2011.

[6] J. Garcia-Aguilar, C. Belmonte, W. D. Wong, S. M. Goldberg, and R. D. Madoff, "Anal fistula surgery. Factors associated with recurrence and incontinence," Diseases of the Colon \& Rectum, vol. 39, no. 7, pp. 723-729, 1996.

[7] B. Li, X. Xu, X. Wang et al., "A systems biology approach to understanding the mechanisms of action of Chinese herbs for treatment of cardiovascular disease," International Journal of Molecular Sciences, vol. 13, no. 12, pp. 13501-13520, 2012.

[8] F. Zhao, L. Guochun, Y. Yang, L. Shi, L. Xu, and L. Yin, “A network pharmacology approach to determine active ingredients and rationality of herb combinations of ModifiedSimiaowan for treatment of gout," Journal of Ethnopharmacology, vol. 168, pp. 1-16, 2015.

[9] Q. Yin, Y. Wei, Z. De, W. Qingming, and Z. Wei, "Effect of different concentration and treatment of Chinese herbal fumigant of Cuyuxunxi prescription on complications after anal fistuletomy," World Traditional Chinese Medicine, vol. 8, no. 12, pp. 1417-1419, 2013.

[10] T. Pei, C. Zheng, C. Huang et al., "Systematic understanding the mechanisms of vitiligo pathogenesis and its treatment by
Qubaibabuqi formula," Journal of Ethnopharmacology, vol. 190, pp. 272-287, 2016.

[11] M. Lyu, C. L. Yan, H. X. Liu et al., "Network pharmacology exploration reveals endothelial inflammation as a common mechanism for stroke and coronary artery disease treatment of Danhong injection," Scientific Reports, vol. 7, no. 1, p. 15427, 2017.

[12] C. Ma, T. Xu, X. Sun et al., "Network pharmacology and bioinformatics approach reveals the therapeutic mechanism of action of baicalein in hepatocellular carcinoma," EvidenceBased Complementary and Alternative Medicine, vol. 2019, Article ID 7518374, 15 pages, 2019.

[13] G. Yu, W. Wang, X. Wang et al., "Network pharmacologybased strategy to investigate pharmacological mechanisms of Zuojinwan for treatment of gastritis," BMC Complementary and Alternative Medicine, vol. 18, no. 1, p. 292, 2018.

[14] J. Ru, P. Li, J. Wang et al., "TCMSP: a database of systems pharmacology for drug discovery from herbal medicines," Journal of Cheminformatics, vol. 6, no. 1, p. 13, 2014.

[15] L. Huang, D. Xie, Y. Yu et al., "TCMID 2.0: a comprehensive resource for TCM," Nucleic Acids Research, vol. 46, no. D1, pp. D1117-D1120, 2018.

[16] D. S. Wishart, Y. D. Feunang, A. C. Guo et al., "DrugBank 5.0: a major update to the DrugBank database for 2018," Nucleic Acids Research, vol. 46, no. D1, pp. D1074-D1082, 2018.

[17] V. Law, C. Knox, Y. Djoumbou et al., "DrugBank 4.0: shedding new light on drug metabolism," Nucleic Acids Research, vol. 42, no. D1, pp. D1091-D1097, 2014.

[18] C. J. Mattingly, M. C. Rosenstein, A. P. Davis, G. T. Colby, J. N. Forrest Jr., and J. L. Boyer, "The comparative toxicogenomics database: a cross-species resource for building chemical-gene interaction networks," Toxicological Sciences, vol. 92, no. 2, pp. 587-595, 2006.

[19] A. P. Davis, C. J. Grondin, R. J. Johnson et al., "The comparative toxicogenomics database: update 2019," Nucleic Acids Research, vol. 47, no. D1, pp. D948-D954, 2019.

[20] A. P. Davis, C. G. Murphy, M. C. Rosenstein, T. C. Wiegers, and C. J. Mattingly, "The comparative toxicogenomics database facilitates identification and understanding of chemical-gene-disease associations: arsenic as a case study," BMC Medical Genomics, vol. 1, no. 1, p. 48, 2008.

[21] D. Szklarczyk, A. L. Gable, D. Lyon et al., "STRING v11: protein-protein association networks with increased coverage, supporting functional discovery in genome-wide experimental datasets," Nucleic Acids Research, vol. 47, no. D1, pp. D607-D613, 2019.

[22] P. Shannon, A. Markiel, O. Ozier et al., "Cytoscape: a software environment for integrated models of biomolecular interaction networks," Genome Research, vol. 13, no. 11, pp. 2498-2504, 2003.

[23] C. T. Keith, A. A. Borisy, and B. R. Stockwell, "Multicomponent therapeutics for networked systems," Nature Reviews Drug Discovery, vol. 4, no. 1, pp. 71-78, 2005.

[24] E. E. Schadt, S. H. Friend, and D. A. Shaywitz, "A network view of disease and compound screening," Nature Reviews Drug Discovery, vol. 8, no. 4, pp. 286-295, 2009.

[25] X. Zhang, J. Gu, L. Cao et al., "Network pharmacology study on the mechanism of traditional Chinese medicine for upper respiratory tract infection," Molecular BioSystems, vol. 10, no. 10, pp. 2517-2525, 2014.

[26] H. Y. Fang, H. W. Zeng, L. M. Lin et al., "A network-based method for mechanistic investigation of Shexiang Baoxin pill's treatment of cardiovascular diseases," Scientific Reports, vol. 7, no. 1, p. 43632, 2017. 
[27] F. Yamashita and M. Hashida, "In silico approaches for predicting ADME properties of drugs," Drug Metabolism and Pharmacokinetics, vol. 19, no. 5, pp. 327-338, 2004.

[28] M. Huber-Lang, A. Kovtun, and A. Ignatius, "The role of complement in trauma and fracture healing," Seminars in Immunology, vol. 25, no. 1, pp. 73-78, 2013.

[29] U. Amara, D. Rittirsch, M. Flierl et al., "Interaction between the coagulation and complement system," in Advances in Experimental Medicine and Biology, vol. 632, pp. 71-79, Springer, New York, NY, USA, 2008.

[30] R. Wiegner, S. Chakraborty, and M. Huber-Lang, "Complement-coagulation crosstalk on cellular and artificial surfaces," Immunobiology, vol. 221, no. 10, pp. 1073-1079, 2016. 


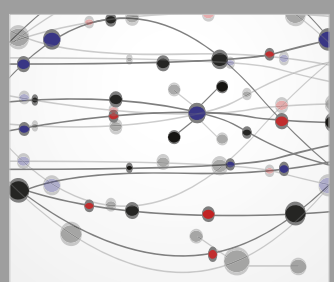

The Scientific World Journal
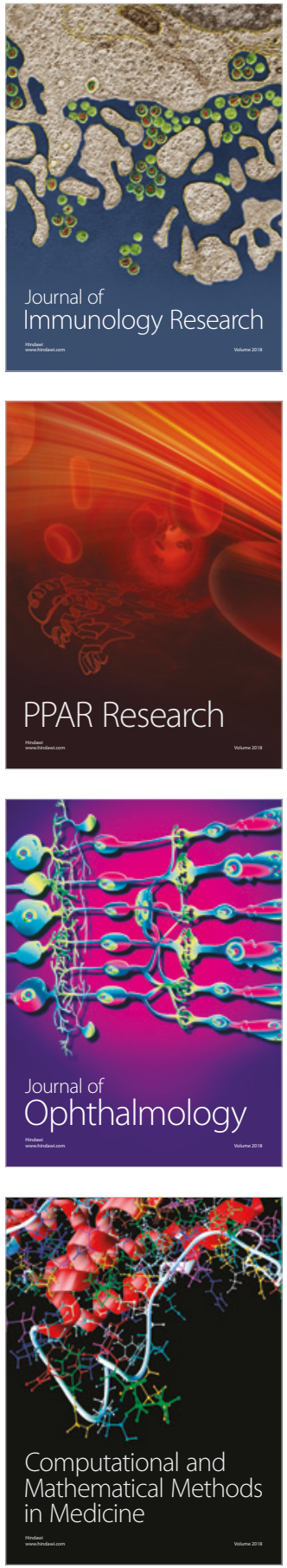

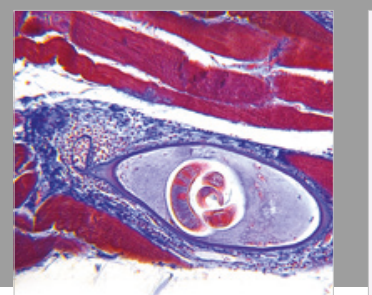

Gastroenterology Research and Practice

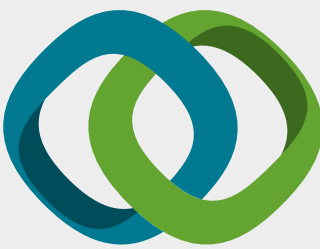

\section{Hindawi}

Submit your manuscripts at

www.hindawi.com
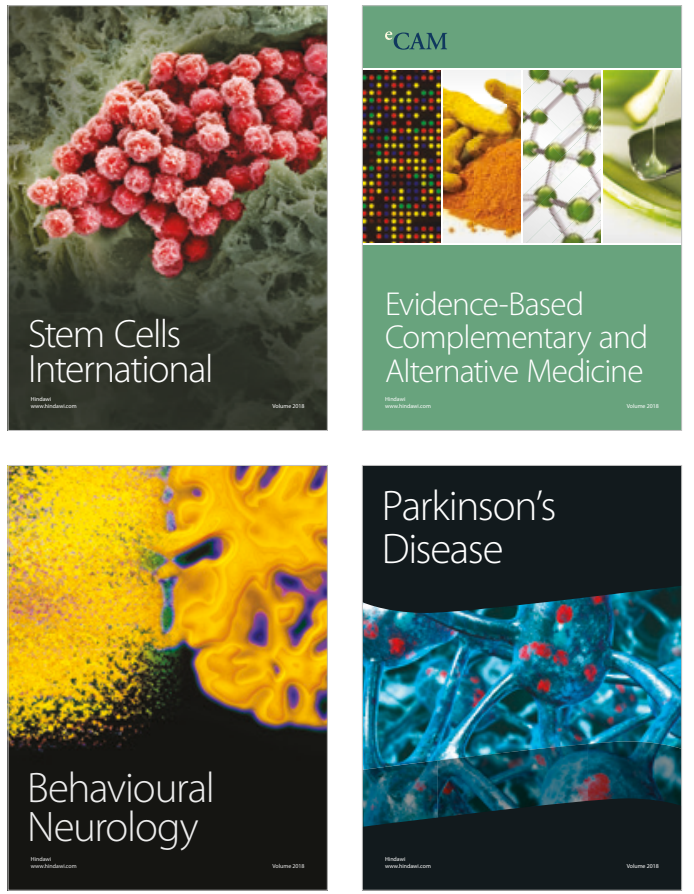

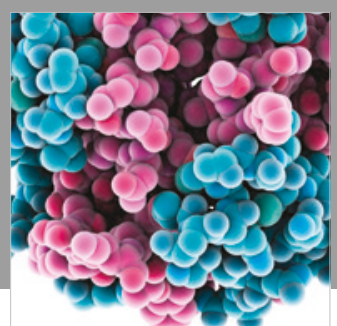

ournal of

Diabetes Research

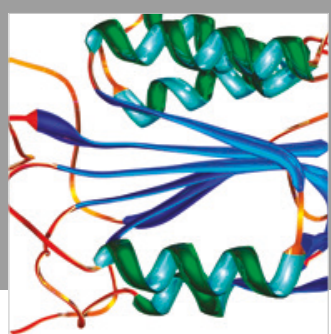

Disease Markers
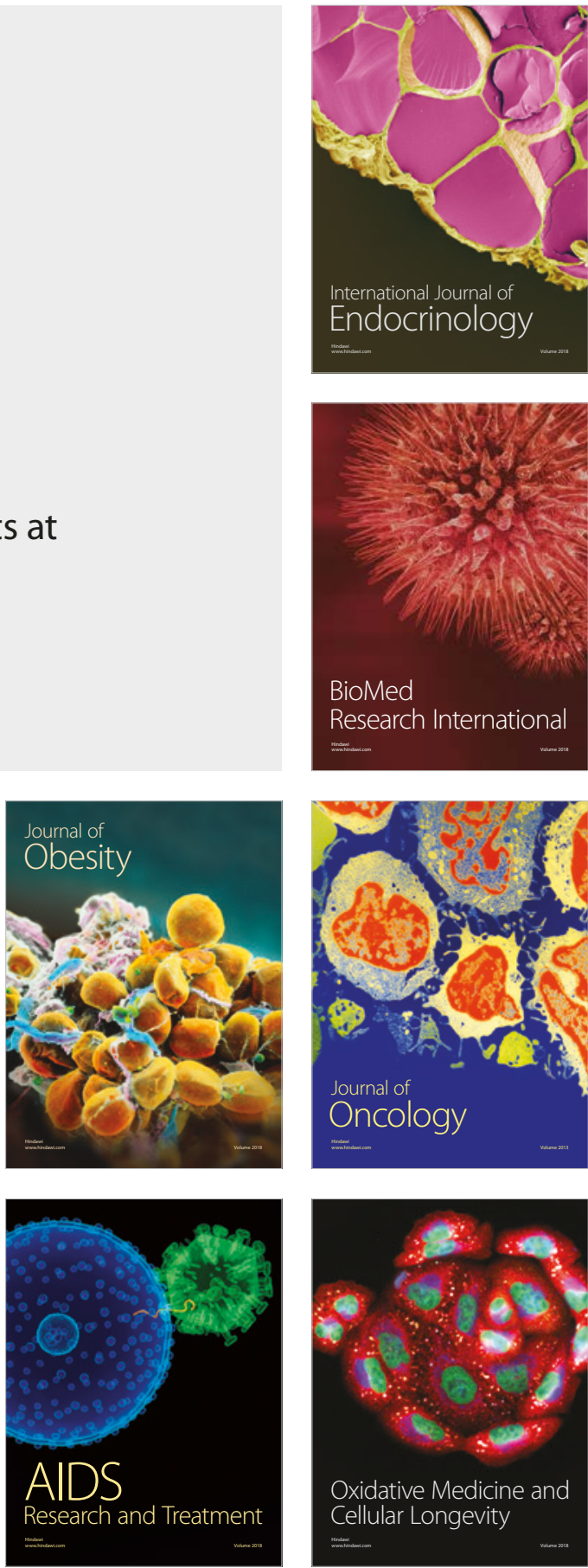